\title{
Behavior Analyses of Phase Boundaries in the Stochastic Mean Curvature Flow by the Level Set Method
}

\author{
Masaaki Ishikawa and Keiichi Miyajima \\ Dept. of Computer Science and Systems Engineering, Yamaguchi University \\ 2-16-1 Tokiwadai, Ube, Yamaguchi 755-8611, Japan \\ E-mail: ishi@yamaguchi-u.ac.jp
}

\begin{abstract}
An influence of a random noise on a mean curvature flow of a phase boundary is investigated. The phase boundary is defined as a curve or a surface separating different physical states such as a water-ice interface. The mean curvature flow is motion that the phase boundary moves with a normal velocity equals the mean curvature at each point on the phase boundary. Since we treat a stochastic mean curvature flow, we formulate it by a stochastic level set equation, which is a nonlinear stochastic partial differential equation with a unique solution in a sense of a stochastic viscosity solution. In numerical simulations, behaviors of the stochastic mean curvature flows in two- and three-dimensional spaces are studied.
\end{abstract}

\section{Introduction}

An analysis of a mean curvature flow is one of important problems in many fields of research including material science and computer vision. For example, the grain boundary in annealing of metal moves according to the mean curvature flow [1]. And the mean curvature flow plays a very important role in computer vision problems like restoration and segmentation in image processing. The level set method [2] is a powerful tool to analyze the mean curvature flow. This method requires to solve the nonlinear partial differential equation, to which notion of a weak solution [3] based on integration by part cannot be applied. So, a viscosity solution [4], one of weak solutions, has been developed based on the maximum principle of the partial differential equation. Since one of our aim is to study the influence of the random noise on the mean curvature flow, we must treat stochastic mean curvature flow. The stochastic mean curvature flow is formulated by a stochastic level set equation and the sufficient condition for existence of the unique stochastic viscosity solution [5]-[8] is shown. In numerical simulations, the mean curvature flows under noise of a star shape in two-dimensional space, axial symmetric barbell and torus shapes in three-dimensional space are investigated. The influence of the random noise on the mean curvature flow is clarified from simulation results.

\section{Stochastic Mean Curvature Flow}

Let $S_{0}$ be a connected boundary of a bounded open set of $R^{n}$. Consider the phase boundary $S(t)$ with $S(0)=S_{0}$ in $R^{n}$, which evolves according to the mean curvature flow, i.e., $S(t)$ moves at the velocity equals to the mean curvature at each point on $S(t)$. As shown in Fig. 1, we assume that the phase boundary $S(t)$ can be represented by a zero level set of a smooth function $u(t, x)$ in such a way that

$$
S(t)=\left\{x \mid u(t, x)=0, x \in R^{n}\right\} .
$$

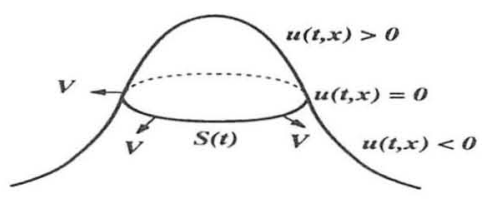

Fig. 1: Normal velocity $V$ of the phase boundary $S(t)$

Then, an outward normal velocity $V$ and the mean curvature [2] at the hypersurface $S(t)$ are given by

$$
V=\frac{u_{t}}{|\operatorname{grad} u|}, \quad \kappa=-\operatorname{div}\left(\frac{\operatorname{grad} u}{|\operatorname{grad} u|}\right)
$$

where $u_{t}$ denotes the partial derivative of $u$ in time $t$.

In the mean curvature flow, $S(t)$ evolves at the normal velocity $V$ related to the mean curvature $\kappa$ as follows

$$
V=-\kappa
$$

Since $S(t)$ is interpreted as a grain boundary [1] in metal, there exist cases where the velocity $V$ contains the fluctuation caused by impurities in metal. So, modeling randomness of such a fluctuation as a white noise, consider

$$
V=-\kappa+b \frac{d w(t)}{d t}
$$

where $b$ is a constant and $w(t)$ is a Wiener process and its formal derivative $d w(t) / d t$ denotes a Gaussian white noise.

From (2) and (4), the stochastic model of the mean curvature flow is derived as

$$
d u(t, x)=|\operatorname{grad} u| \operatorname{div}\left(\frac{\operatorname{grad} u}{|\operatorname{grad} u|}\right) d t
$$




$$
+b|\operatorname{grad} u| \circ d w(t), \quad(t, x) \in \Theta \times R^{n}
$$

with an initial condition

$$
u(0, x)=u_{0}(x), \quad x \in R^{n}
$$

where $\Theta=(0, T),(T$ is a positive constant) and the symbol $\circ$ of the 2 nd term of the R.H.S. of (5) denotes a stochastic integral of Stratonovich type [9].

Remark 2.1. If the stochastic integrals in (5) is not Stratonovich type but Ito one [9], we cannot prove the existence of the unique stochastic viscosity solution of (5), because any function $\beta(u)$ of $u$ does not satisfy the same equation (5) as $u$ due to generation of an additional term related to variance of $w(t)$.

Lions and Souganidis [5]-[8] proved that the nonlinear stochastic partial differential equation below had a unique solution in the sense of the stochastic viscosity solution;

$$
d u=F\left(D^{2} u, D u\right) d t+H(D u) \circ d w, \text { in } \Theta \times R^{n}
$$

with the initial condition

$$
u(0)=u_{0} \text {, on } R^{n}
$$

where $D u=\operatorname{grad} u$ and $D^{2} u$ denotes the Hessian matrix of second derivatives of $u$.

Since the stochastic level set equation (5) can be written as (7), it follows from results in [5]-[8] that the following theorem holds.

Theorem 2.1 If $u_{0} \in U B C\left(R^{n}\right)$, there exists a unique stochastic viscosity solution of (5) belongs to $U B C\left(R^{n}\right)$, where $U B C\left(R^{n}\right)$ denotes the space of uniformly bounded continuous functions on $R^{n}$.

For the proof, see [5]-[8].

\section{Simulations}

In this section, numerical simulations of the mean curvature flow of some phase boundaries are performed by a level set method [2]. So, we begin with the explanation of the level set method.

\subsection{Level Set Method}

The level set method is stated as below;

(Step-1) For a given initial phase boundary $S(0)$, generate the level function $u_{0}(x)$ which satisfies

$$
S(0)=\left\{x \mid u_{0}(x)=0, x \in R^{n}\right\} .
$$

(Step-2) Solve the following stochastic level set equation of the mean curvature flow under the initial data $u_{0}(x)$ generated at Step-1;

$$
d u=|\operatorname{grad} u| \operatorname{div}\left(\frac{\operatorname{grad} u}{|\operatorname{grad} u|}\right) d t+b|\operatorname{grad} u| \circ d w(t)
$$

(Step-3) From the solution $u(t, x)$ of $(10)$, the position of the phase boundary $S(t)$ at time $t$ is determined by

$$
S(t)=\left\{x \mid u(t, x)=0, x \in R^{n}\right\} .
$$

\subsection{Mean Curvature Flow in 2D-space}

In this section, consider the mean curvature flow of the horseshoe shape as shown in Fig. 2(a). Figure 2(b) is the initial level function $u_{0}(x)$ with the zero level set as Fig. 2(a). Figure 3 shows time evolution of the mean curvature flow of the horseshoe shape under the noise and the no noise. In Fig. 3, dotted and solid lines correspond to the mean curvature flow under the noise and the no noise. From Fig. 3, we can see that the horseshoe shape becomes convex one and shrinks with time and motion of the mean curvature flow under the noise is slower than one under the no noise.

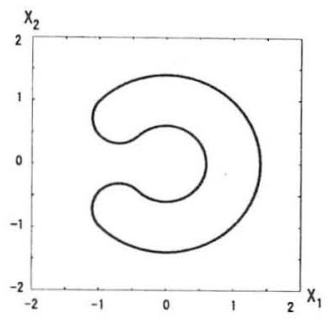

(a) Initial curve

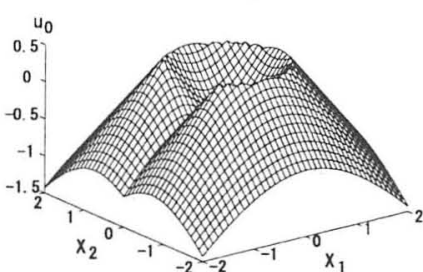

(b) Initial level function $u_{0}(x)$
Fig. 2: Horseshoe shape
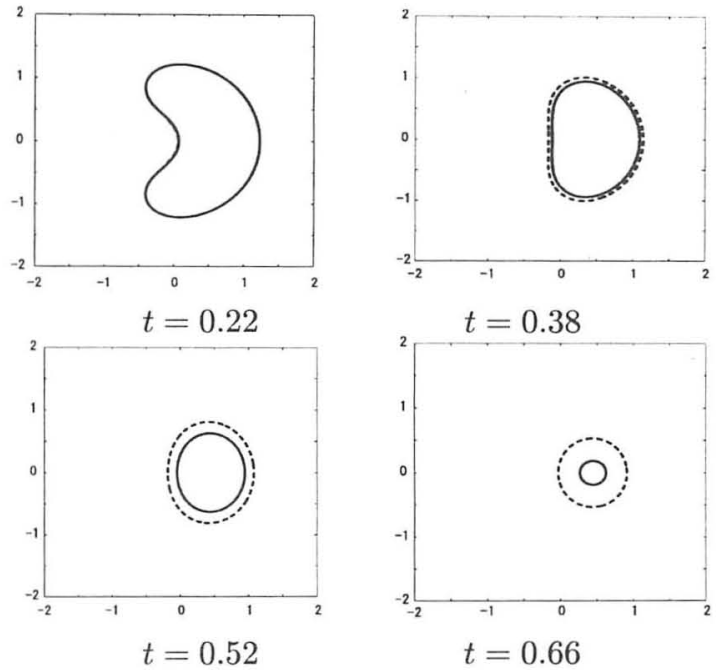

Fig. 3: Mean curvature flow of horseshoe shape under no noise(solid line) and noise(dotted line)

\subsection{Mean Curvature Flow in 3D-space}

\subsubsection{Mean Curvature Flow of Barbell Shape}

In this section, consider the mean curvature flow of the surface $S_{q}(0)$ of the axial symmetric barbell shape as shown in Fig. 4, which is described by

$$
\begin{aligned}
S_{q}(0)=\left\{(x, y, z) ; r^{2}\right. & =\left(1-x^{2}\right)\left(1-q+q x^{2}\right)^{2}, \\
r^{2} & \left.=y^{2}+z^{2},|x| \leq 1\right\}
\end{aligned}
$$

where $0 \leq q \leq 1$ and $S_{0}(0)$ becomes a unit sphere.

Changing the parameter $q$ in (12) as $q=0.65$ and 0.8 , simulations are performed. 

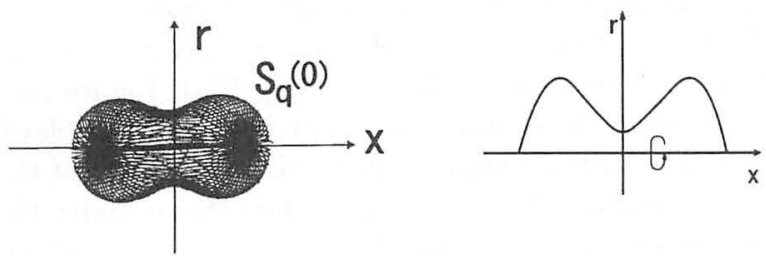

Fig. 4: Axial symmetric barbell shape

【 Case-1: Barbell shape with $q=0.65 】$ Figure 5 shows the initial level functions $u_{0}(x)$ and its zero level set, which is the cross section of the barbell shape with $q=0.65$ at plane includes $x$-axis. Figures 6 and 7 show time evolution of cross section of the barbell shape with $q=0.65$. Simulations in Figs. 6 and 7 are performed under the same initial condition but the noise sequences are different. In Fig. 6, the barbells first become convex and annihilate in both of the noise and the no noise cases and the barbell in the no noise case annihilates earlier than one in the noise case. On the other hand, in Fig. 7 , the barbell in the noise case separates into two convex shapes at the end of simulation and annihilates, so that the barbell in the noise case annihilates earlier than the no noise case.

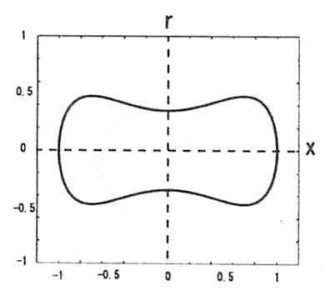

(a) Section view

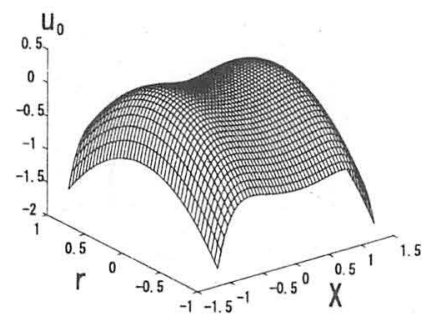

(b) Initial level function $u_{0}(x)$
Fig. 5: Section view of the barbell with $q=0.65$ and the initial level function $u_{0}(x)$
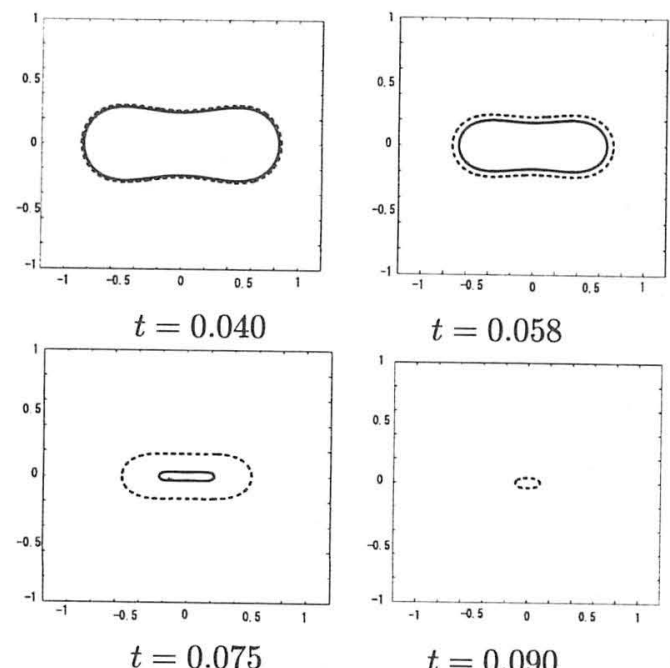

Fig. 6: Mean curvature flow I of the barbell with $q=$ 0.65 under no noise(solid line) and noise(dotted line)
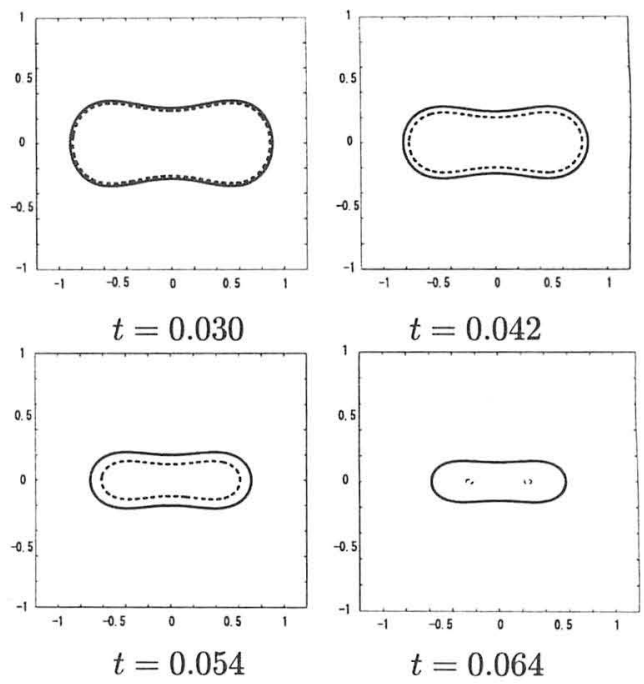

Fig. 7: Mean curvature flow II of the barbell with $q=$ 0.65 under no noise(solid line) and noise(dotted line)

【 Case-2: Barbell shape with $q=0.8 \rrbracket$ Figure 8 shows the cross section of the barbell shape with $q=0.8$ at plane includes $x$-axis and the initial level functions $u_{0}(x)$ with Fig. 8(a) as its zero level set. Figure 9 is the cross section of time evolution of mean curvature flow of the barbell shape with $q=0.8$ and dotted and solid curves depict motion of the barbell shape under the noise and under the no noise. We can see that there arises split of the barbell because of the difference of mean curvatures between the central part and rightand-left parts. And there is no clear difference between the noise and the no noise cases at the beginning, however, there is clear difference between them as the end of the simulation, i.e., and the curve under the no noise annihilates earlier than one under the noise.

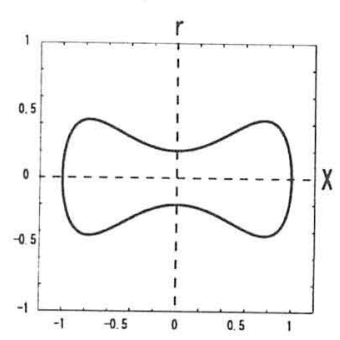

(a) Section view

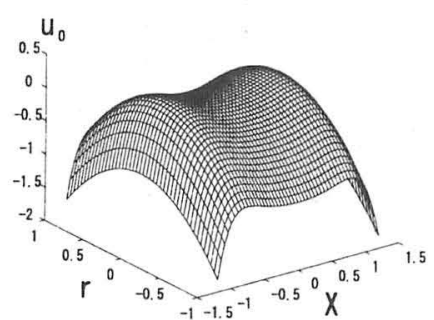

(b) Initial level function $u_{0}(x)$
Fig. 8: Barbell shape with $q=0.8$

\subsubsection{Mean Curvature Flow of Torus Shapes}

Consider two types of axial symmetric torus shapes as shown in Fig. 10, which are parameterized by $r_{1}$ 

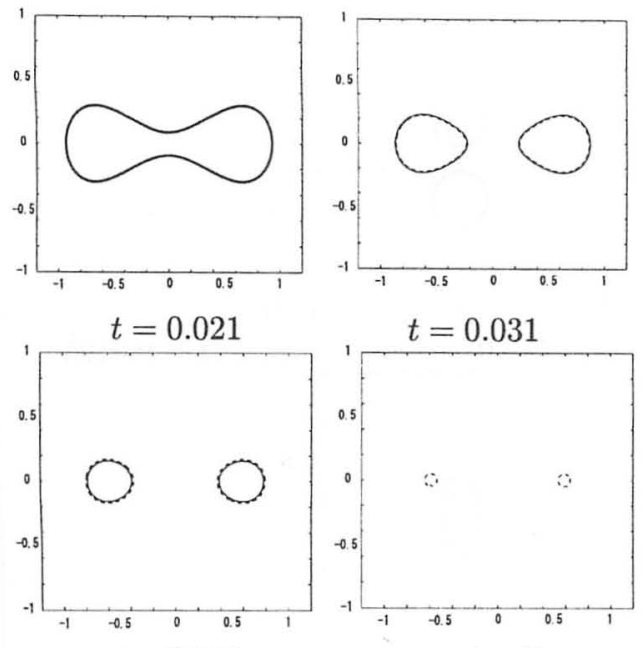

$t=0.039$

$t=0.047$

Fig. 9: Mean curvature flow of barbell with $q=0.8$ under no noise(solid line) and noise(dotted line)

and $r_{2}$ as in Fig. 11. Figure 11 shows the cross section of the torus shape at plane includes axis of revolution and its elevation view. Torus shapes I and II have the same value of $r_{1}=0.6$ and different value of $r_{2}$, i.e., $r_{2}=0.25$ and $r_{2}=0.5$ respectively.

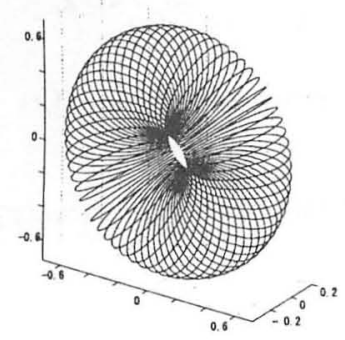

(a) Torus I

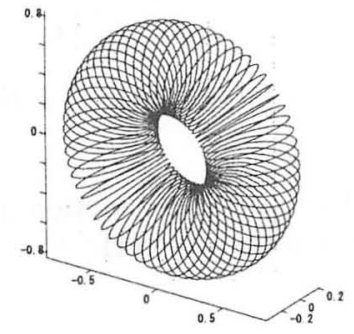

(b) Torus II

$\left(r_{1}=0.6, r_{2}=0.5\right)$ $\left(r_{1}=0.6, r_{2}=0.25\right)$

Fig. 10: Two types of torus shapes

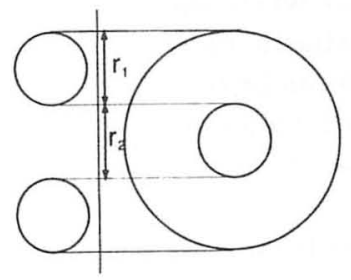

Fig. 11: Parameters in cross section of torus and its elevation view
【Case-3: Torus shape I 】 Figure 12 shows the initial level function $u_{0}(x)$ and its zero level set. The zero level set in Fig. 12(a) coincides with the cross section of the torus shape I in Fig. 10(a). The time evolution of the mean curvature flow of the torus shape I of Fig. 10(a) are shown in Fig. 13 under the no noise case, and results under two different noise sequences of the same torus shape I are shown in Figs. 14 and 15. Figure 16 is the time evolution of cross sections of the torus shapes of Figs. 13 to 15. From Fig. 16, we can see that the torus becomes the sphere and annihilates under the no noise. But under the noise, there exist two types of evolution, one is that the torus becomes the sphere and then annihilates as the no noise case, and the other is that the torus keeps its shape and annihilates. In the case of becoming the sphere, the phase boundary under the no noise annihilates earlier than one under the noise as shown in Figs. 13, 14 and 16. On the other hand, in the case of keeping the torus shape by the noise as shown in Figs. 15 and 16, the phase boundary under the noise annihilates faster than the case under no noise because the mean curvature of the torus is higher than one of sphere at the same time.

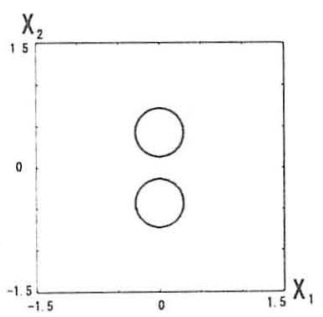

(a) Initial curve

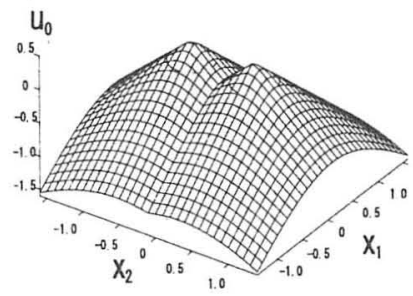

(b) Initial level function $u_{0}(x)$
Fig. 12: Initial value for the torus I

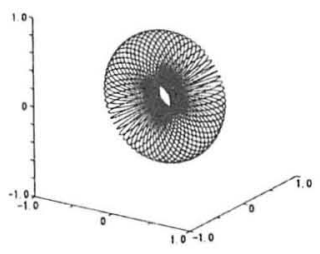

$t=0$

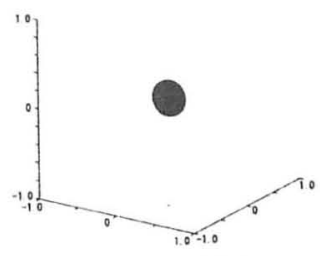

$t=0.0625$

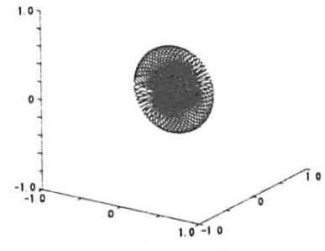

$t=0.0375$

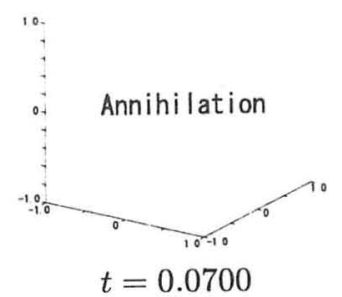

Fig. 13: Mean curvature flow of the torus I under no noise 

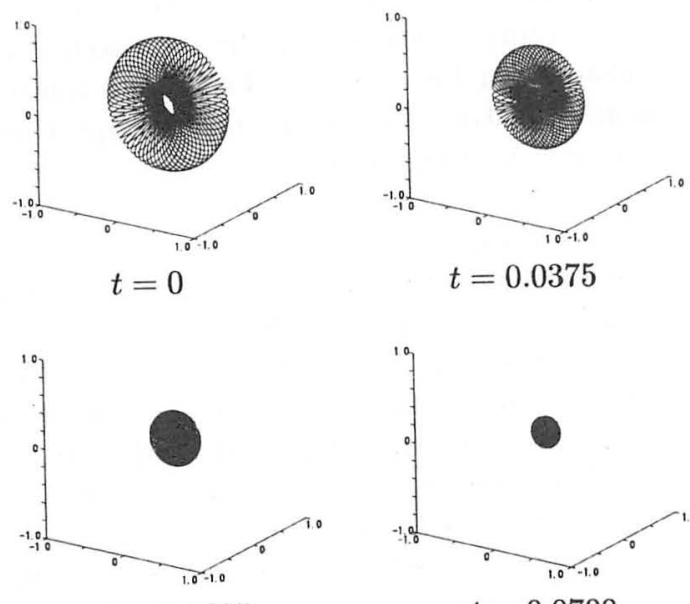

$t=0.0625$

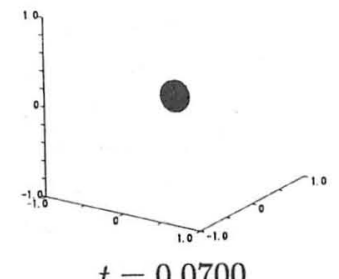

Fig. 14: Mean curvature flow I of the torus I under noise

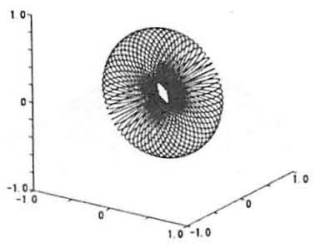

$t=0$

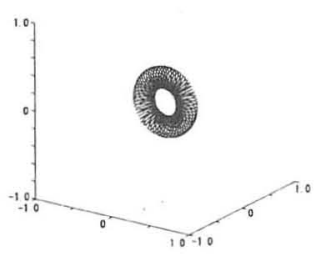

$t=0.0375$

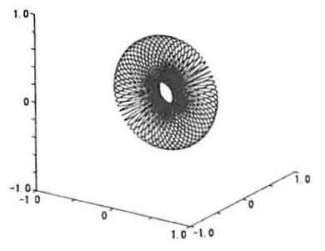

$t=0.0125$

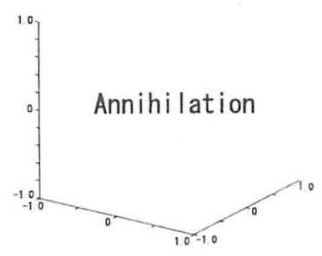

$t=0.0625$
Fig. 15: Mean curvature flow II of the torus I under noise

【Case-4: Torus shape II 】Figure 17 is the initial level function $u_{0}(x)$ which gives the cross section of the torus shape II in Fig. 10(b) as its zero level set. The time evolution of the mean curvature flow of the torus shape II under the no noise and the noise are shown in Figs. 18 and 19. Figure 20 is cross sections of the torus shapes in Figs. 18 and 19. And the meaning of dotted and solid curves is same as in Fig. 16. From Figs. 18 to 20, we can see that the torus keeps its shape and annihilates in both of the noise and the no noise cases and the phase boundary under the no noise annihilates earlier than one under the noise.
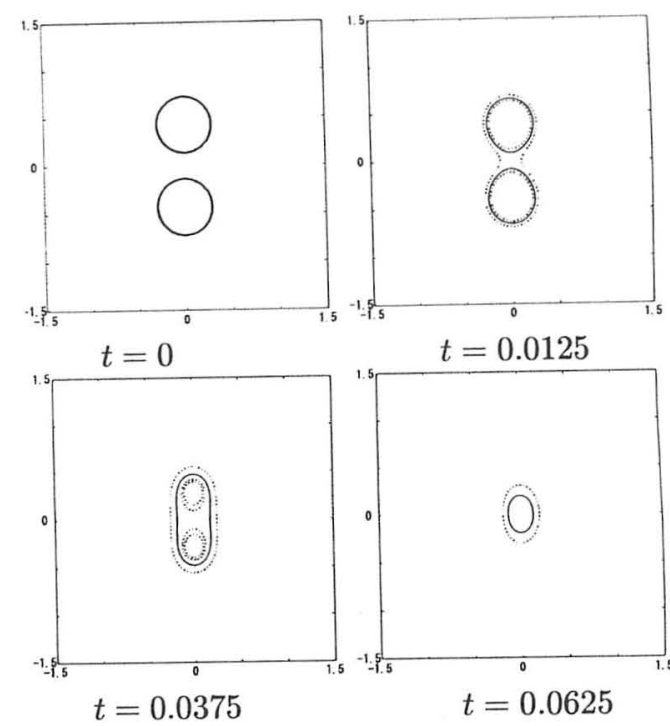

Fig. 16: Cross section of mean curvature flow of torus I under noise(dotted curve) and no noise(solid curve)

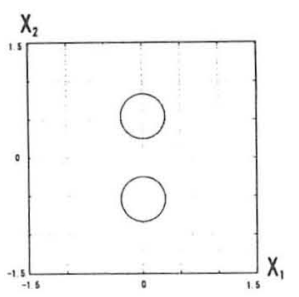

(a) Cross section

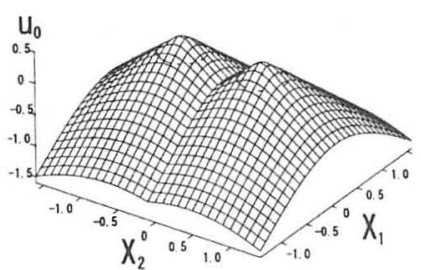

(b) Initial level function $u_{0}(x)$
Fig. 17: Cross section and initial level function $u_{0}(x)$ for torus shape II

\section{Conclusions}

By the level set method, the behavior of the mean curvature flows of axial symmetric barbell and torus shapes and the influence of the random noise on the mean curvature flows have been studied. Although the stochastic mean curvature flows of some barbell and torus shapes do not have the fattening property for each sample process, we have shown numerically that there exists a case where sample behaviors of the phase boundary in stochastic mean curvature flows of barbell and torus shapes can be topologically different from the deterministic ones under the same initial condition by the random noise. From the simulation results, we can see that

(i) the sample behaviors of the phase boundaries of some barbell and torus shapes under the same initial conditions can topologically change in the stochastic case by the random noise despite of the uniqueness of the solution because the uniqueness does not guarantee that each sample process is the same one. 


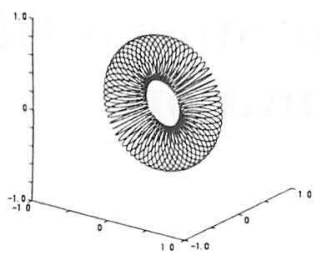

$t=0.02$

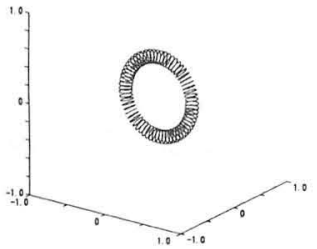

$t=0.045$

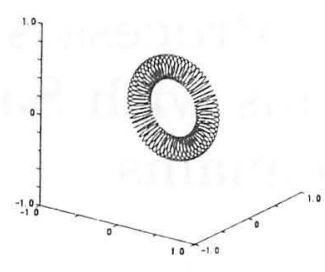

$t=0.04$

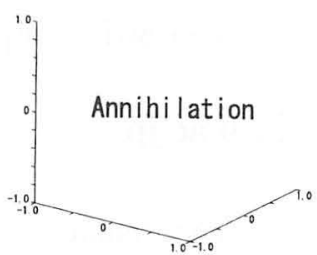

$t=0.05$
Fig. 18: Mean curvature flow of torus II under no noise

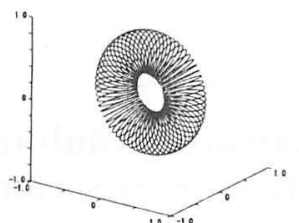

$t=0.020$

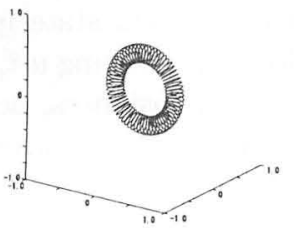

$t=0.045$

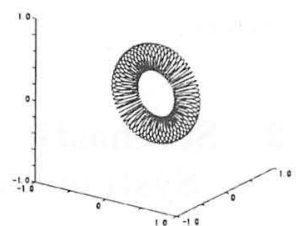

$t=0.040$

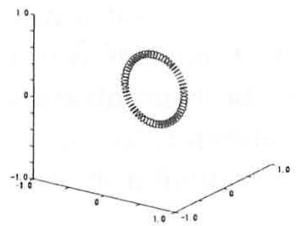

$t=0.050$
Fig. 19: Mean curvature flow of torus II under noise

(ii) in the case where the topological change of the phase boundary occurs by the random noise, annihilation in the stochastic case is much faster than the deterministic one because the curvature of the phase boundary becomes much higher than one in the deterministic case at the same time by the topological change of the phase boundary.

A part of this work is supported by The Ministry of Education, Culture, Sports, Science and Technology under Grant-in-Aid for Research (C)(2)14550217.

\section{References}

[1] M. E. Gurtin, "Thermomechanics of evolving phase boundaries in the plane," Clarendon Press, Oxford, 1993.

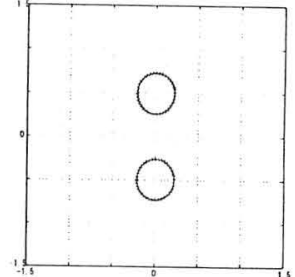

$t=0.020$

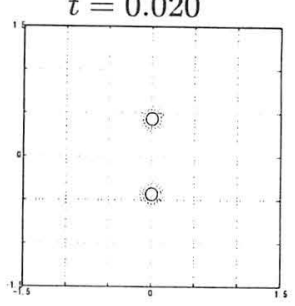

$t=0.045$

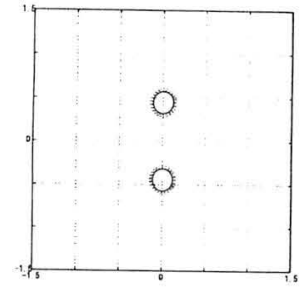

$t=0.040$

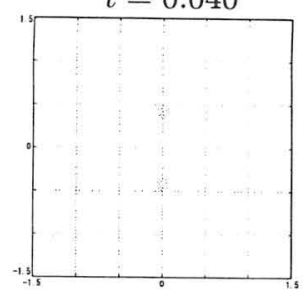

$t=0.050$
Fig. 20: Cross section of mean curvature flow of torus II under noise(dotted curve) and no noise(solid curve)

[2] S. Osher and R. Fedkiw, "Level set methods and dynamic implicit surfaces," Applied Mathematical Sciences 153, Springer-Verlag, 2003.

[3] J. L. lions, " Optimal control of systems governed by partial differential equations," Springer-Verlag, 1971.

[4] M. G. Crandall, H. Ishii and P. L. Lions, "User's guide to viscosity solutions of second order partial differential equations," Bull. Amer. Math. Soc., vol. 27, pp.1-67, 1992.

[5] P. L. Lions and P. E. Souganidis, "Uniqueness of weak solutions of fully nonlinear stochastic partial differential equations," Comptes RendusAcademie des Science Paris, SERIE 1, vol. 331, pp.783-790, 2000.

[6] P. L. Lions and P. E. Souganidis, "Fully nonlinear stochastic pde with semilinear stochastic dependence Lions," Comptes Rendus-Academie des Science Paris, SERIE 1, vol.331, pp.617-624, 2000.

[7] P. L. Lions and P. E. Souganidis, "Fully nonlinear stochastic partial differential equations: nonsmooth equations and applications," Comptes Rendus-Academie des Science Paris, SERIE 1, vol.327, pp.735-742, 1998.

[8] P. L. Lions and P. E. Souganidis, "Fully nonlinear stochastic partial differential equations," Comptes Rendus-Academie des Science Paris, SERIE 1, vol.326, 1085-1092, 1998.

[9] H. Kunita, "Stochastic flows and stochastic differential equations," Cambridge University Press, Cambridge, 1990. 